\title{
Ocular Surface Alterations and Topical Antiglaucomatous Therapy: A Review
}

\author{
Alessandro G. Actis* and Teresa Rolle \\ University of Torino - Department of Surgical Sciences - Eye Clinic, Via Juvarra 19 - 10121 - Torino, Italy
}

\begin{abstract}
Ocular Surface Disease (OSD) is prevalent among medically treated patients with glaucoma. This is basically related to three key-points: OSD and glaucoma are both prevalent in elderly and are common comorbidities in the same patient; the role of the active ingredient of the medical antiglaucomatous therapy; the role of the preservative agent of this medical therapy.

Considering the actual state of literature we can state that the active glaucoma agent have a role in OSD, but the main cause seems to be the preservative agent, in particular referring to benzalkonium chloride, BAK.

In the clinical evaluation of dry eye patients there is no actually established gold standard. Since the ocular surface injury not only causes dry eye, red eye, eye itching, photophobia and other discomforts, but also increases the risk of failure of glaucoma surgery in patients, it becomes fundamental a complete and good clinical evaluation of OSD (considering Schirmer's test, tear breakup time, corneal and conjunctival staining) together with a good evaluation of patients' quality of life (with validated questionnaires).

Development of complex preparations, preservative-free and/or novel preservative preparations for glaucoma therapy could provide a promising approach in the prevention of ocular surface injuries.
\end{abstract}

Keywords: Benzalkonium chloride (BAK), dry eye, glaucoma, ocular surface disease (OSD), quality of life (QoL).

\section{INTRODUCTION}

Primary open angle glaucoma (POAG) is a progressive neurodegeneration of retinal ganglion cells (RCGs) and their axons characterized by a specific pattern of visual field and optic nerve head damage $[1,2]$. Clinical trials confirmed the importance of intraocular pressure (IOP) in the development and progression of open-angle glaucoma, even if evidence suggests the existence of ocular and systemic factors, in addition to IOP, that can be responsible of this development and progression. IOP is still the only risk factor that we can effectively treat with medical and surgical therapy $[3,4]$.

Tear film deficiencies are among the most common eye problems [5]: epidemiologic studies have reported that more than $6 \%$ of the population over the age of 40 suffer from dry eye, with the prevalence increasing to $15 \%$ of the population over the age of 65 [6-8]. Using a prevalence of $6 \%$ and the 2000 census data, there are an estimated 7.1 million people in the US over the age of 40 who experience dry eye symptoms. Most studies have found an increasing prevalence with age and some studies have shown a greater prevalence of dry eye among women [9-11].

The 2007 International Dry Eye WorkShop (DEWS) defined dry eye as a multifactorial disorder of the tear film and ocular surface that results in eye discomfort, visual

*Address correspondence to this author at the University of Torino Department of Surgical Sciences - Eye Clinic, Via Juvarra 19, 10121, Torino, Italy; Tel: + 39011 5666085; Fax: +39 011 539024;

E-mail: alessandro.actis@gmail.com disturbance, and often ocular surface damage, characterized by hyperosmolarity of tear film and inflammation of ocular surface [12].

Dry Eye Disease (DED) - associated to Ocular Surface Disease (OSD) - can be described as a disorder involving multiple interacting mechanisms $[12,13]$. Dysfunction of any lacrima function unit component can lead to DED by causing alterations in the volume, composition, distribution, and/or clearance of the tear film. Two mutually reinforcing mechanisms, tear hyperosmolarity and tear film instability, have been identified [12]. Tear hyperosmolarity can arise from both low aqueous flow or excessive evaporation. Hyperosmolar tears can damage ocular surface epithelium by activating an inflammatory cascade. While acute inflammation may initially be accompanied by increased reflex tearing and blinking, chronic inflammation may result in reduced corneal sensation and decreased reflex activity, leading to increased evaporation and tear film instability. Inflammation can also result in goblet cell loss and decreased mucin production, which further contributes to tear film instability. Tear film instability can arise secondary to hyperosmolarity, or can be the initiating event, as in lipid layer abnormalities following meibomian gland disease. Tear film instability results in increased evaporation, which contributes to tear hyperosmolarity. Regardless of the initiating event or etiology, inflammation is usually a key factor in perpetuating DED [14]. Chronic DED may result in further pathologic changes. For example, patients with moderate to severe DED may develop reversible squamous metaplasia and punctate erosions of the ocular surface epithelium [15]. DED is also 
the most common cause of filamentary keratitis, a condition characterized by strands of degenerated epithelial cells and mucus attached to the cornea. Friction between the filaments and the eyelid during blinking can result in further epithelial damage, inflammation, and filament formation. Filamentary keratitis often becomes chronic, and is a common finding in severe DED [16, 17]. Rarely, severe DED may lead to complications such as ocular surface keratinization, microbial keratitis, corneal neovascularization, ulceration, perforation, and scarring and finally severe vision loss [18].

\section{DISCUSSION}

Ocular Surface Disease and DED are prevalent among medically treated patients with glaucoma. Patients with glaucoma and ocular hypertension suffer OSD at a higher prevalence rate than patients without these ocular conditions [19].

This is related basically to three concepts:

1. OSD and glaucoma are both prevalent in elderly and are common comorbidities in the same patient [20]. OSD has an age-dependent prevalence, affecting approximately $11 \%$ of patients between the ages of 40 and 59 and $18 \%$ of those older than age 80 [21]. The prevalence of POAG also increases with age.The glaucoma risk increasing with the age was noticed in almost all population studies [4]. Tsai [22] concluded that $66 \%$ of subjects with severe OSD also have glaucoma.

2. The role of the active ingredient of the medical therapy.

3. The role of the preservative agent (particularly benzalkonium chloride, BAK) of the medical therapy.

We already explained the close relationship between age and both POAG and OSD. It is mandatory to better explain the role of the glaucoma therapy.

\section{The Role of the Active Ingredient of the Medical Therapy}

The benefit of IOP reduction in the treatment of glaucoma has been confirmed in large controlled, prospective clinical trials. Medical treatment is predominantly used as first line therapy and therefore the majority of patients receive several decades of treatment. The main topical hypotensive medications include timolol (non-selective adrenergic beta-blocker), carteolol (non-selective adrenergic beta-blocker with intrinsic sympathomimetic activity), carbonic anhydrase inhibitors as dorzolamide or brinzolamide, brimonidine (adrenergic agonist alpha-2 selective), prostaglandin analogues (latanoprost, travoprost, bimatoprost, tafluprost). Prostaglandin analogues and beta-blockers are the most commonly prescribed first-line therapies [1, 2]. Timolol, introduced in 1978, in a few years [23] became the most widely used drug in the treatment of glaucoma since it was approved for general use. Despite the major contraindications (asthma, history of obstructive pulmonary disease, sinus bradycardia, heart block or cardiac failure) today it is one of the most used drugs and is also available in fixed-dose combinations. Already in 1979 Nielsen and Eriksen [24] underlined how in 64 patients treated with timolol eye drops, seven patients developed transitory sensation of dry eyes. Two of these subjects also had xerostomia. In all these seven patients a reduction of Schirmer test and break-up time was noted. Fasina et al. [25] evaluated the effect of Timolol maleate on tear film break-up time in a Nigerian population. 192 eyes of 96 subjects were examined in a hospital based case-control study after being administered pre-coded questionnaires.There was significant difference in the mean break-up time of cases (10.45 secs) and controls ( 30.18 secs)

Preservative-free timolol solution has a favourable effect on the tear turnover of patients with glaucoma and ocular hypertension in comparison with timolol containing BAK [26], even if the integrity of the precorneal tear film persisted to be affected when using timolol without BAK [26, 27]. Timolol without preservative can be recommended in those patients who have keratoconjunctivitis sicca or a borderline tear production in order not to increase the severity of the dry eye.

In 1996 Latanoprost, the first prostaglandin agent, was introduced in medical therapy, followed by Travoprost and Bimatoprost in 2001 and Tafluprost in 2008. Unoprostone was introduced in some countries in 2000. Prostaglandins and their combination drugs have become the top-line choices for treatment in the past decade because of the oncedaily dosing and great efficacy in lowering IOP. Different papers in literature demonstrates how switching the therapy from a preserved to a preservative-free prostaglandin preparation in topical glaucoma medication can lead to a great improve of OSD.

Generally there is no sufficient evidence to demonstrate that the active glaucoma agent can lead to OSD. Blondin et al. [28] demonstrated that carteolol, timolol, betaxolol or latanoprost did not activate complement system, an early mediator of the inflammatory response. Furthermore timolol and betaxolol exerted an anti-inflammatory effect by preventing complement activation.

Pisella et al. [29] even suggested a potential protective effect of the prostaglandin analogue and, to a lesser extent, timolol against the toxicity of BAK in conjunctival cells. A recent multicenter, international, non-interventional study enrolled 448 patients with glaucoma or ocular hypertension in therapy with one ore more topical IOP-lowering medications (on average 1.9 IOP-lowering medications). OSD was highly prevalent in the population studied. The worsening of OSD symptoms and score (measured with Ocular Surface Disease Index, OSDI) was significantly related to longer duration since diagnosis and higher number of medications [30]. Valente et al. [31] published a recent study to evaluate the presence of symptoms of tear film dysfunction by using the Ocular Surface Disease Index (OSDI) questionnaire in fifty glaucomatous patients and to examine whether they have ocular surface signs. Fifty-two percent of patients in therapy with preserved antiglaucomatous drops showed symptoms of tear film dysfunction. Signs of ocular surface diseases seemed to be greater in patients under $>2$ medications. Symptoms correlated to signs only in patients in monotherapy with $\beta$ blockers drops. Considering the actual state of literature we can conclude that the active glaucoma agent can have a role in OSD, but the main cause seems to be the preservative agent [19]. 


\section{The Role of the Preservative Agent of the Medical Therapy}

The need for sterility in multidose eyedrops has led to the inclusion of an antimicrobial preservative in these solutions. Commonly actually used preservatives in antiglaucomatous therapy are BAK, Purite ${ }^{\mathbb{R}}$ (Allergan Inc., Irvine, California, USA), Polyquaternum 1 as Polyquad ${ }^{\circledR}$ (Alcon Research, Fort Worth, Texas, USA), SofZia ${ }^{\circledR}$ (Novartis AG, Basel, Switzerland).

Other preservatives include organo-mercurial derivatives (such as thimerosal) that precipitate bacterial proteins and are active against Gram-positive bacteria and nonsporulating organisms; amidines (such as chlorhexidine) that damage the cytoplasmatic membrane and are active particularly on Gram-positive bacteria and cocci; alcohols (such as chlorobutanol) that can cross the bacterial lipid layer (bacteriostatic and antimicotic activity); parabens (the ester of parahydroxybenzoic acid) that target fungi and finally the oxychlorinated complexes which disrupt cells function.

The most frequently used preservative, benzalkonium chloride (BAK), has consistently demonstrated its toxic effects in laboratory, experimental, and clinical studies. As a quaternary ammoniums act mainly with a detergent activity, which dissolve bacterial walls and membranes and damage the cytoplasmatic contents. Nenciu et al. [32] carried out a 40 week, prospective, randomized trial enrolling 18 patients divided in four groups according to their therapy: betablockers, prostaglandin analogues, carbonic anhydrase inhibitors. The morphometric analysis of histological sections of conjunctiva revealed squamous metaplasia, inflammation and fibrosis. Also the immunohistochemistry confirmed the tendency to chronic inflammations. These findings pointed to BAK as a major causal factor of conjunctival metaplasia. The group of Baudouin confirmed histopathologic effects of antiglaucomatous drugs on the conjunctiva and showed similar effects in the trabecular meshwork with human and animal studies [33]. This experimental studies showed that BAK is for a large part responsible for these toxic or immunoinflammatory effects on the ocular structures. As a quaternary ammonium BAK has been shown to cause tear film instability, loss of goblet cells, conjunctival squamous metaplasia and apoptosis, disruption of the corneal epithelium barrier, and damage to deeper ocular tissues. The mechanisms causing these effects have not been fully elucidated, although the involvement of immunoinflammatory reactions with the release of proinflammatory cytokines, apoptosis, oxidative stress, as well as direct interactions with the lipid components of the tear film and cell membranes have been well established [34]. Lot of researches have been conducted to discover and develop less toxic preservative than BAK and quaternary ammoniums, but only a few have been proposed and are commercially available, since a preservative must be a potent antimicrobial agent while not being cytotoxic [34].

Noecker et al. [35] investigated in rabbit eyes the effect of Purite ${ }^{\circledR}$, a stabilized oxychloro complex. Although the adverse effects of glaucoma medications on the ocular surface are likely multifactorial, 1-month treatment with glaucoma medications containing higher levels of benzalkonium chloride (BAK) resulted in greater corneal damage and conjunctival cell infiltration than medications preserved with Purite ${ }^{\circledR}$ or with lower levels of BAK.

Polyquad $^{\circledR}$ is a polyquaternum, a family of polycationic polymers that are used in personal care industries. Polyquaternum 1 (as Polyquad $^{\circledR}, \mathrm{PQ}$ ) is commonly used in ophthalmology. Liang $\mathrm{H}$ et al. [36] evaluated in rabbits a new formulation of travoprost $0.004 \%$ ophthalmic solution preserved with $\mathrm{PQ}$, with commercially available formulations of BAK-preserved travoprost $0.004 \%$ ophthalmic solution and BAK-preserved latanoprost $0.005 \%$ ophthalmic solution, suggesting a greater safety advantage for the ocular surface of patients receiving chronic glaucoma treatment with PQ-preserved drugs. Considering Labbé et al. [37] PQ, except in high concentration of $0.5 \%$, did not create important changes in ocular surface compared to saline solution, whereas BAK induced major toxic effects at mild to high concentrations with destruction of globet cells.

SofZia ${ }^{\circledR}$ is a new preservative generation that functions as a microbicidal agent through oxidative properties. This ionic buffer solution, comprised of borate, propylene glycol, sorbitol, and zinc chloride, converts to nontoxic byproducts after contact with ocular surface cations [38]. A prospective randomized multicentre single-masked comparative study [39] enrolled 220 patients with open-angle glaucoma or ocular hypertension who had been treated with BAKpreserved latanoprost $0.005 \%$ monotherapy for at least 3 months. After a switch to SofZia-preserved travoprost $0.004 \%$ the study indicated that frequency of keratoconjunctival epitheliopathy significantly decreased in the travoprost SofZia-preserved group $(\mathrm{p}=0.036)$ and the intergroup difference was also significant $(\mathrm{p}=0.001)$. SPK scores and TBUT were significantly improved in the travoprost group $(\mathrm{p}=0.034,0.049)$, also with significant intergroup differences in the cornea excluding the inferior area and TBUT. There were no significant intergroup differences in changes of the hyperaemia scores and the IOP reduction.

Ammar [40] investigated the potential cytotoxicity of various topical ophthalmic glaucoma formulations containing different preservatives in cultured human trabecular meshwork (TM) and non-pigmented ciliary epithelial (NPCE) cell lines. They demonstrated that substitution of BAK from topical ophthalmic drugs (compared to SofZia ${ }^{\circledR}$ and Polyquad ${ }^{\circledR}$ ) results in greater viability of cultured TM cells, the cells involved in the conventional outflow pathway. Cultured NPCE, responsible for aqueous inflow, appear more resilient to BAK. Anyway BAK enhances all characteristics of TM degeneration typical of glaucoma, i.e. trabecular apoptosis, oxidative stress, induction of inflammatory chemokines. This causes degeneration in acute experimental conditions, potentially mimicking long-term accumulation. BAK was also shown to access the TM after repeated instillations. These findings support the hypothesis that preservatives may cause further long-term trabecular degeneration and therefore enhance outflow resistance, reducing the impact of IOP-lowering agents [41].

We can conclude that BAK is the most commonly used preservative in ophthalmology and is more toxic than other or newer preservatives [42]. Substitution of BAK with Polyquad $^{\circledR}$, Purite $^{\circledR}$ or sofZia ${ }^{\circledR}$ resulted in significantly higher 
percentages of live conjunctival and corneal cells. Further studies are needed to understand the role upon TM cells and the clinical implications of these findings [43].

Totally preservative-free topical medication can be suggested for patients with ocular surface disease, frequent eye drop administration, proven allergy to preservatives and contact lens wear [43]. Improvements in OSD and quality of life have been demonstrated in several studies switching to preservative free drugs, i.e. timolol, prostaglandin agents, timolol/dorzolamide fixed combination, prostaglandin/ timolol fixed combination [44-48].

We also have to underline that there is a large evidence from clinical and experimental studies that long-term use of topical drugs may induce, as a consequence of chronic ocular surface changes, the potential risk of failure for further glaucoma surgery. Subclinical inflammation has also been widely described in patients receiving antiglaucoma treatments for long periods of time, with inflammatory cell infiltration and fibroblast activation in the conjunctiva and subconjunctival space. The preservative, especially BAK, could induce or enhance such inflammatory changes. Postoperatively, the ocular surface also plays an important role, as the conjunctiva interacts with aqueous humor and subconjunctival fibrosis may block aqueous outflow and cause surgical failure [49].

Martone et al. [50] ruled an in vivo confocal microscopy analysis of effects of topical antiglaucoma therapy with preservative on corneal innervation and morphology. The density of superficial epithelial cells was reduced in all glaucomatous patients, except for the preservative-free group. On the contrary, the density of basal epithelial cells of glaucomatous preservative therapy groups was higher than control and preservative-free groups. Stromal keratocyte activation and the number of beads were higher in all glaucoma preservative groups. The number of sub-basal nerves was lower in all glaucoma groups than in the control group and tortuosity was significantly higher in glaucoma than control groups. Confocal microscopy can be an important diagnostic tool in the evaluation of dry eye disease, with possible therapeutical implications [51, 52] as switching topical therapy.

\section{Clinical Practice}

In clinical practice the ideal medical therapy for glaucoma to prevent OSD (and to improve adherence) follows some basic statements.

1. The introduction of BAK-free or even preservative free agents in common use is a great advancement in glaucoma therapy, considering that this therapy is a continuous one.

2. The severity of OSD symptoms is positively correlated to the number of IOP-lowering medication used [53]. It's important to underline that medical therapy has improved over the past decade through the introduction of more powerful topical drugs with long-lasting effects. These drugs make it possible to control IOP in many glaucoma patients with a once daily dose of a single agent. However, in some patients in whom IOP is not sufficiently controlled with monotherapy, adjunctive therapy is required. In such patients it is possible to use a fixed combination of glaucoma drugs, two IOP lowering agents combined in a single formulation. That's why the introduction of combination therapies can be considered an important advancement in glaucoma therapy [54].

3. A complete and good clinical evaluation of OSD should be periodically performed. In the clinical evaluation of dry eye patients there is no actually established gold standard: several tests are used in the clinical practice which analyze patient's symptoms, tear production, tear quality, ocular surface damage. All of these diagnostic test have low sensibility and little reproducibility, in particulary in patients with severe dry eye. Tear film hyperosmolarity is recognized as an important pathogenetic factor in dry eye syndrome, but its use is currently limited due to the complexity in its measurement $[12,55]$. The same is true for confocal microscopy, a promising very good diagnostic tool with poor clinical practice yet $[50,55]$. Arita R. et al. evaluated also how lid margin abnormality, superficial punctate keratopathy and meibum scores were higher in glaucoma patients under therapy, concluding how long-term use of antiglaucoma eye drops was associated with alterations in meibomian gland morphology and function [56].

Considering these statements it becomes fundamental to evaluate periodically:

- $\quad$ Schirmer test score

- $\quad$ tear breakup time score with with fluorescein $1 \%$

- corneal staining with fluorescein $1 \%$ and a validate grading scale (i.e. National Eye Institute (N.E.I.) / Industry Workshop Scale; Oxford Grading Scale; SICCA ocular staining score) [57-59]

- $\quad$ conjunctival staining preferably with lissamine green and a validate grading scale (i.e. National Eye Institute (N.E.I.) / Industry Workshop Scale; Oxford Grading Scale; SICCA ocular staining score) [57-59]

- the quality of life with a validate questionnaire (i.e. OSDI questionnaires; Ocular Comfort Index (OCI)/ Ocular Symptom Scale (OSS) questionnaires) [60, 61]

A recent paper underlines the importance of the assessment of Quality of Life (QoL). [62]. This crosssectional study has been ruled with OSDI score and with Glaucoma Quality of Life-15/GQL15 [62] and demonstrated that OSD is more common in patients with increasing glaucoma severity and is associated with poorer glaucomarelated QoL and higher exposure to BAK.

It is fundamental to evaluate QoL with a validate method, i.e. the National Eye Institute Visual Function Questionnaire (NEI-VFQ) or the Ocular Surface Disease Index (OSDI).

Abnormal OSDI was found in a large number of glaucomatous and ocular hypertensive patients, even if large proportion of patients with severe OSDI has got normal or a mild to moderate alteration of clinical test. OSDI increases with the greater the number of glaucoma drugs prescribed. A large proportion of patients with severe OSDI had normal or 
a mild to moderate alteration of clinical tests. Also psychological and environmental factors or the severity and awareness of the disease can influence OSDI [63].

Li M et al. [64] underlined in a recent paper how visionrelated QoL in dry eye patients was impaired and was correlated with anxiety and depression, further implicating this condition as an important public health problem deserving increased attention and resources.

\section{CONCLUSION}

Patients with topically treated glaucoma present OSD more often than a similar control group [65]. The presence of DED negatively influences the patient's QOL. The patients with glaucoma's ocular surface status should be evaluated regularly to ensure the timely detection and treatment of pathologic signs on the ocular surface.

The ocular surface injury not only causes dry eyes, red eye, eye itching, photophobia and other discomforts, but also increases the risk of failure of glaucoma surgery in patients [66].

The commonly used preservative Benzalkonium Chloride (BAK) plays an important role in ocular surface damage and its side effects are dose- and time-dependent, particularly, in the combined medications. Good periodically clinical evaluation and prevention of ocular injury should be seriously taken into consideration in the anti-glaucoma medical treatment. Development of complex preparations, preservative-free and/or novel preservative preparations for glaucoma therapy could provide a promising approach in the prevention of ocular surface injury $[52,55,66-68]$.

\section{CONFLICT OF INTEREST}

The authors confirm that this article content has no conflict of interest.

\section{ACKNOWLEDGEMENTS}

Declared none.

\section{REFERENCES}

[1] Tuulonen A, Airaksinen PJ, Erola E, et al. The finish evidencebased guideline for open angle glaucoma. Acta Ophthalm Scand 2003; 81: 3-18.

[2] European Glaucoma Society. Terminology and Guidelines for Glaucoma. $3^{\text {rd }}$ Edition. Dogma Editrice. Savona (Italia) 2008.

[3] Bron A, Chaine G, Villain M, et al. Les facteurs de risque du glaucome primitif à angle ouvert. J Fr Ophth 2008; 31(4): 435-44.

[4] Actis AG, Penna R, Brogliatti B, et al. An internal medicine perspective of risk factors for primary open-angle glaucoma (POAG) progression: Torino's study. Minerva Oftalmologica 2012; 54(3): 85-92.

[5] McGinnigle S, Naroo SA, Eperjesi F. Evaluation of dry eye. Surv Ophthalmol 2012; 57(4): 293-316.

[6] McCarty CA, Bansal AK, Livingston PM, et al. The epidemiology of dry eye in Melbourne, Australia. Ophthalmology 1998; 105: 1114-19.

[7] Bjerrum KB. Keratoconjunctivitis sicca and primary Sjiogren's syndrome in a Danish population aged 30-60 years. Acta Ophthalmol Scand 1997; 75: 281-6.

[8] Schein OD, Munoz B, Tielsch JM, et al. Prevalence of dry eye among the elderly. Am J Ophthalmol 1997; 124: 723-28.

[9] McCarty CA, Bansal AK, Livingston PM, et al. The epidemiology of dry eye in Melbourne, Australia. Ophthalmology 1998; 105: 1114-19.

[10] Schein OD, Munoz B, Tielsch JM, et al. Prevalence of dry eye among the elderly. Am J Ophthalmol 1997; 124: 723-28.
[11] Moss SE, Klein R, Klein BE. Prevalence of and risk factors for dry eye syndrome. Arch Ophthalmol 2000; 118(9): 1264-68.

[12] Research in dry eye: report of the Research Subcommittee of the International Dry Eye WorkShop (2007). Ocular Surf 2007; 5(2): 179-93.

[13] Tavares Fde P, Fernandes RS, Bernardes TF, et al. Dry eye disease. Semin Ophthalmol 2010; 25(3): 84-93.

[14] Behrens A, Doyle JJ, Stern L, et al. Dysfunctional tear syndrome: a Delphi approach to treatment recommendations. Cornea 2006; 25: 900-907.

[15] Perry HD. Preferred practice pattern: dry eye syndrome. Am J Manag Care 2008; 14: S79- S87. Avaliable online at: http://www. aao.org/ education/guidelines/ppp/upload/Dry_Eye Syndrome-2.pdf

[16] Diller R, Sant S. A case report and review of filamentary keratitis. Optometry 2005; 76: 30-36.

[17] Albietz J, Sanfilippo P, Troutbeck R, et al. Management of filamentary keratitis associated with aqueous- deficient dry eye. Optom Vis Sci 2003; 80: 420-30.

[18] Labbé A, Brignole-Baudouin F, Baudouin C. Ocular surface investigations in dry eye. J Fr Ophthalmol 2007; 30(1): 76-97

[19] Stewart WC, Stewart JA, Nelson LA. Ocular surface disease in patients with ocular hypertension and glaucoma. Curr Eye Res. 2011; 36(5): 391-98.

[20] Fechtner RD, Godfrey DG, Budenz D, et al. Prevalence of ocular surface complaints in patients with glaucoma using topical intraocular pressure-lowering medications. Cornea. 2010; 29(6): 618-21.

[21] Moss SE, Klein R, Klein BE. Prevalence of and risk factors for dry eye syndrome.Arch Ophthalmol 2000; 118: 1264-68.

[22] Tsai JH, Derby E, Holland EJ, et al. Incidence and prevalence of glaucoma in severe ocular surface disease. Cornea2006; 25(5) 530-2.

[23] Remis LL, Epstein DL. Treatment of glaucoma. Annu Rev Med 1984; 35: 195-205

[24] Nielsen NV, Eriksen JS. Timolol transitory manifestations of dry eyes in long term treatment. Acta Ophthalmol (Copenh). 1979; 57(3): 418-24

[25] Fasina O, Ashaye AO, Ajayi BG. The effect of timolol maleate on tear film break-up time in Nigerians. Afr J Med Med Sci 2008; 37(1): 43-7.

[26] Kuppens EV, de Jong CA, Stolwijk TR, et al. Effect of timolol with and without preservative on the basal tear turnover in glaucoma. Br J Ophthalmol 1995; 79(4): 339-42.

[27] de Jong C, Stolwijk T, Kuppens E, et al. Topical timolol with and without benzalkonium chloride: epithelial permeability and autofluorescence of the cornea in glaucoma. Graefes Arch Clin Exp Ophthalmol. 1994; 232(4): 221-4

[28] Blondin C, Hamard P, Cholley B, et al. In vitro effects of preserved or preservative-free antiglaucoma medications on human complement system. Curr Eye Res 2003; 27: 253-59

[29] Pisella PJ, Debbasch C, Hamard P, et al. Conjunctival proinflammatory and proapoptotic effects of latanoprost and preserved and unpreserved timolol: an ex vivo and in vitro study. Invest Ophthalmol Vis Sci 2004; 45: 1360-68.

[30] Garcia-Feijoo J, Sampaolesi JR. A multicenter evaluation of ocular surface disease prevalence in patients with glaucoma. Clin Ophthalmol 2012; 6: 441-6.

[31] Valente C, Iester M, Corsi E, et al. Symptoms and signs of tear film dysfunction in glaucomatous patients. J Ocul Pharmacol Ther 2011; 27(3): 281-5.

[32] Nenciu A, Stefan C, Ardelean C. Structural and immunihistochemical changes of conjunctuiva induced by topical antiglaucoma medication. Oftalmologia 2004; 48(1): 35-42.

[33] Baudouin C, Pisella PJ, Fillacier K, et al. Ocular surface inflammatory changes induced by topical antiglaucoma drugs: human and animal studies. Ophthalmology 1999; 106(3): 556-63.

[34] Baudouin C, Labbé A, Liang H, et al. Preservatives in eyedrops: the good, the bad and the ugly. Prog Retin Eye Res 2010; 29(4): 312-34. Epub 2010 Mar 17.

[35] Noecker RJ, Herrygers LA, Anwaruddin R. Corneal and conjunctival changes caused by commonly used glaucoma medications. Cornea 2004; 23(5): 490-6.

[36] Liang H, Brignole-Baudouin F, Riancho L, et al. Reduced in vivo ocular surface toxicity with polyquad-preserved travoprost versus benzalkonium-preserved travoprost or latanoprost ophthalmic solutions. Ophthalmic Res 2012; 48(2): 89-101. 
[37] Labbé A, Pauly A, Liang H, et al. Comparison of toxicological profiles of benzalkonium chloride and polyquaternium-1: an experimental study. J Ocul Pharmacol Ther 2006; 22(4): 267-78.

[38] Anwar Z, Wellik SR, Galor A. Glaucoma therapy and ocular surface disease: current literature and recommendations. Curr Opin Ophthalmol 2013; 24(2): 136-43.

[39] Aihara M, Oshima H, Araie M; EXTraKT study group. Effects of SofZia-preserved travoprost and benzalkonium chloride-preserved latanoprost on the ocular surface -- a multicentre randomized single-masked study. Acta Ophthalmol 2013; 91(1): e7-14.

[40] Ammar DA, Kahook MY. Effects of glaucoma medications and preservatives on cultured human trabecular meshwork and nonpigmented ciliary epithelial cell lines. Br J Ophthalmol 2011; 95(10): 1466-9.

[41] Baudouin C, Denoyer A, Desbenoit N, et al. In vitro and in vivo experimental studies on trabecular meshwork degeneration induced by benzalkonium chloride (an American Ophthalmological Society thesis). Trans Am Ophthalmol Soc 2012; 110: 40-63.

[42] Messmer EM. Preservatives in ophthalmology. Ophthalmologe 2012; 109(11): 1064-70.

[43] Ammar DA, Noecker RJ, Kahook MY. Effects of benzalkonium chloride-preserved, polyquad-preserved, and sofZia-preserved topical glaucoma medications on human ocular epithelial cells. Adv Ther 2010; 27(11): 837-45.

[44] Frezzotti P, Fogagnolo P, Haka G, et al. In vivo confocal microscopy of conjunctiva in preservative-free timolol $0.1 \%$ gel formulation therapy for glaucoma. Acta Ophthalmol 2014; 92(2): e133-40.

[45] Sanford M. Preservative-free latanoprost eye drops in patients with primary open-angle glaucoma/ocular hypertension. Clin Drug Investig 2014; 34(7): 521-8

[46] Abegão Pinto L, Vandewalle E, Gerlier L, Stalmans I. CosoptUD Switch Study Group. Improvement in glaucoma patient quality of life by therapy switch to preservative-free timolol/dorzolamide fixed combination. Ophthalmologica 2014; 231(3): 166-71.

[47] Holló G, Hommer A, Antón López A, Ropo A. Efficacy, safety, and tolerability of preservative-free fixed combination of tafluprost $0.0015 \% /$ timolol $0.5 \%$ versus concomitant use of the ingredients. $\mathrm{J}$ Ocul Pharmacol Ther 2014; 30(6): 468-75.

[48] Inoue K. Managing adverse effects of glaucoma medications. Clin Ophthalmol 2014; 8: 903-13.

[49] Baudouin C. Ocular surface and external filtration surgery: mutual relationships. Dev Ophthalmol 2012; 50: 64-78.

[50] Martone G, Frezzotti P, Tosi GM, et al. An in vivo confocal microscopy analysis of effects of topical antiglaucoma therapy with preservative on corneal innervation and morphology. Am J Ophthalmol 2009; 147(4): 725-35.

[51] Villani E, Magnani F, Viola F, et al. In Vivo Confocal Evaluation of the Ocular Surface Morpho-Functional Unit in Dry Eye. Optom Vis Sci 2013; 90(6): 576-86.
[52] Aydin Kurna S, Acikgoz S, Altun A, et al. The effects of topical antiglaucoma drugs as monotherapy on the ocular surface: a prospective study. J Ophthalmol 2014; 2014: 460-83.

[53] Leung EW, Medeiros FA, Weinreb RN. Prevalence of ocular surface disease in glaucoma patients. J Glaucoma 2008;17: 350-55.

[54] Topouzis F. Combination therapies for glaucoma. Ocular Surgery News Eur/Asia-Pac Ed. January 2009

[55] Lemp AM, Sullivan BD, Crews LA. Biomarkers in dry eye disease. Eur Ophthal Rev 2012; 6(3): 157-63.

[56] Arita R, Itoh K, Maeda S, et al. Comparison of the long term effects of various topical antiglaucoma medications on meibomian glands. Cornea 2012; 31(11): 1229-34

[57] Soni A, Kuo A, Silk W, et al. Comparability and reproducibility of evaluation of ocular surface staining in dry eye patients. Invest Ophthalmol Vis Sci 2004; 45: E-abstract 89.

[58] Yoon KC, Im SK, Kim HG, et al. Usefulness of double vital staining with $1 \%$ fluorescein and $1 \%$ lissamine green in patients with dry eye syndrome. Cornea 2011; 30(9): 972-6.

[59] Bron AJ, Evans VE, Smith JA. Grading of corneal and conjunctival staining in the context of other dry eye tests. Cornea 2003; 22(7): 640-50.

[60] Johnson ME, Murphy PJ. Measurement of ocular surface irritation on a linear interval scale with the ocular comfort index. Invest Ophthalmol Vis Sci 2007; 48(10): 4451-8.

[61] Schiffman RM, Christianson MD, Jacobsen G, et al. Reliability and validity of the ocular surface disease index. Arch Ophthalmol 2000; 118(5): 615-21.

[62] Skalicky SE, Goldberg I, McCluskey P. Ocular surface disease and quality of life in patients with glaucoma. Am J Ophthalmol 2012; 153(1): 1-9.e2.

[63] Nelson P, Aspinall P, Papasouliotis O, et al. Quality of life in glaucoma and its relationship with visual function. J Glaucoma 2003; 12(2): 139-50

[64] Li M, Gong L, Chapin WJ, Zhu M. Assessment of vision-related quality of life in dry eye patients.Invest Ophthalmol Vis Sci 2012; 53(9): 5722-7.

[65] Rossi GC, Tinelli C, Pasinetti GM, et al. Dry eye syndrome-related quality of life in glaucoma patients. Eur J Ophthalmol 2009; 19(4): 572-9.

[66] Villani E, Baudouin C, Efron N, et al. In vivo confocal microscopy of the ocular surface: from bench to bedside. Curr Eye Res 2014; 39(3): 213-31.

[67] Ouyang PB, Duan XC. Ocular surface injury resulted from topical anti-glaucoma medications: prevention and cure. Zhonghua Yan Ke Za Zhi 2012; 48(6): 557-61.

[68] Gomes B, Turiel PR, Marques FP, et al. Signs and symptoms of ocular surface disease in patients on topical intraocular pressurelowering therapy. Arq Bras Opthalmol 2013; 76(5): 282-7.

(C) Actis and Rolle; Licensee Bentham Open.

This is an open access article licensed under the terms of the Creative Commons Attribution Non-Commercial License (http://creativecommons.org/licenses/by-nc/3.0/) which permits unrestricted, non-commercial use, distribution and reproduction in any medium, provided the work is properly cited. 